\title{
Extended DBP for (m,k)-Firm Based QoS
}

\author{
Jiming Chen ${ }^{1}$, Zhi Wang ${ }^{1}$, Yeqiong Song ${ }^{2}$, and Youxian Sun ${ }^{1}$ \\ 1 National Laboratory of Industrial Control Technology, \\ Zhejiang Univ., Hangzhou 310027, P.R.China \\ \{jmchen, wangzhi, yxsun\}@iipc.zju.edu.cn \\ 2 LORIA - TRIO Campus Scientifique B.P. 239 \\ 54506 VANDOEUVRE-les-NANCY, France \\ \{song\}@loria.fr
}

\begin{abstract}
In this paper, an extended DBP (E_DBP) scheme is studied for $(\mathrm{m}, \mathrm{k})$-firm constraint. The basic idea of the proposed algorithm takes into account the distance to exit a failure state, which is a symmetrical notion of distance to fall into a failure state in DBP. Quality of Service (QoS) in terms of dynamic failure and delay is evaluated. Simulation results reveal the effectiveness of E_DBP to provide better QoS.
\end{abstract}

\section{Introduction}

Real-time media servers for delivering audio/video streams need to service hundreds and, possibly, thousands of applications, each with its own quality of service (QoS) requirements. Many such applications can tolerate the loss of a certain fraction of the information requird from the server, resulting in little or no noticeable degradation in QoS [1] 2. Consequently, loss-rate is an important performance measure for the QoS to many real-time media applications. We define the term loss-rate as the fraction of packets in a stream either discarded or serviced later than their delay constraints allow (Deadline) [3].

One of the problems with using loss-rate as a performance metric is that it does not describe when losses are allowed to occur. For most loss-tolerant applications, there is usually a restriction on the number of consecutive packet losses that are acceptable. For example, losing a series of consecutive packets from an audio stream might result in the loss of a complete section of audio, rather than merely a reduction in the signal-to-noise ratio.

A suitable performance metric in this case is a window-based loss-rate, i.e. loss-rate constrained over a finite range, or window, of any consecutive packets. More precisely, an application might tolerate at most k-m packet losses for every $\mathrm{k}$ arrivals at the various service points across a network. Any service discipline attempting to meet these requirements must ensure that the number of violations to the loss-tolerance specification is minimized (if not zero) across the whole stream. In another way, it is same meaning that at least $\mathrm{m}$ packets must be serviced before their deadline in any consecutive k packets. We refer to such QoS requirement as $(\mathrm{m}, \mathrm{k})$-firm constraint. If less than $\mathrm{m}$ packets are serviced successfully in any window $\mathrm{k}$, it is said the application experiences a dynamic failure and 
the current state is called as failure state. An approach called Distance-based Priority (DBP) based on $(\mathrm{m}, \mathrm{k})$-firm idea has been proposed to schedule multiple packet streams competing for service on a single server each having its own $(\mathrm{m}, \mathrm{k})$-firm constraints 4 . It has been showed in that when streams have same or different $(\mathrm{m}, \mathrm{k})$-firm constraint requirement and are identical (i.e. with same packet transmission time distribution, same packet inter-arrival distribution and same deadline distribution), DBP is especially more beneficial to tightening the probability of dynamic failure than conventional scheduling scheme where all packets are serviced at the same priority level [4]. This idea is then generalized under the name weakly hard real-time to deal with real-time applications that allows some packet losses without violating the desired behaviors of application [5. In this paper, we proposed an extended DBP (E_DBP) scheme to study $(\mathrm{m}, \mathrm{k})$-firm based QoS, and compared the E_DBP and DBP for streams with $(\mathrm{m}, \mathrm{k})$-firm in terms of probability of dynamic failure and delay.

The rest of paper is structured as follows. Section 2 describes DBP scheme and some relative work. In section 3, E_DBP scheduling is proposed. In section 4, performance metric of QoS about dynamic failure and delay is evaluated through simulation in overloaded scenarios. Finally, we make some concluding remarks.

\section{DBP Scheduling on Steams with $(\mathrm{m}, \mathrm{k})$-Firm}

We begin this section by defining the problem that we focus on real-time streams with $(\mathrm{m}, \mathrm{k})$-firm . Then, how the DBP scheme works is described. The drawbacks are stated in the last.

\subsection{Problem Definition}

In order to define the real-time scheduling problem based on $(\mathrm{m}, \mathrm{k})$-firm constraint addressed as part of this paper, we introduce the following definitions:

Application Model. As briefly mentioned in the introduction section, DBP scheduling is designed to study how to efficiently serve multiple streams under $(\mathrm{m}, \mathrm{k})$-firm constraints sharing a single server. This system is called multiple input queues on single server (MIQSS). This model can be used to study a large category of computer and telecommunication systems such as multiple tasks executing in a $\mathrm{CPU}$, transmission of packets issued from multiple packet sources sharing a same transmission medium or network interconnection equipment (switch or router). The proposed model is for $n$ loss-tolerant applications generating $n$ packet streams $\tau_{i}(i=1,2, \ldots, n)$ that will be served by a single server. Each stream is formed by a source and a waiting queue, where a packet issued from a source waits until being chosen by the server. The server chooses packets at the head of queues according to its scheduling scheme.

In such a model, scheduling scheme is of prime importance to provide not only $(\mathrm{m}, \mathrm{k})$-firm guarantee for each individual stream (end user's point of view) but also good server utilization (system designer's point of view). 
Stream Characterization. A stream $\tau_{i}$ is characterized by a 3 -tuple $\left(C_{i}, D_{i}, T_{i}\right)$, where $C_{i}$ is the service time for a packet in stream $\tau_{i}$. There, it is assumed that all packets in $\tau_{i}$ have the same service time.For the purposes of this paper, where time is divided into fixed-sized slots, each and every packet can be serviced in one such slot. Deadline $D_{i}$ is the latest time a packet finishes its service. If a packet cannot be finished by $D_{i}$, it will be discarded, which is called deadline miss or packet loss. $T_{i}$ is the inter-arrival time between consecutive packets.

Loss-Tolerance. This is specified by $(\mathrm{m}, \mathrm{k})$-firm constraint, where $\mathrm{m}$ is the least number of the packets that should be transmitted successfully by their deadline for any window $\mathrm{k}$ of consecutive packet arrivals in the same stream. Otherwise, a stream experiences a dynamic failure. The rate at which a stream experiences dynamic failure is therefore a measure of how often the QoS falls below the acceptable level, which is defined as the probability of dynamic failure.

Problem Statement. The problem addressed in this paper is to propose a more effective scheduling scheme than DBP to guarantee better $(\mathrm{m}, \mathrm{k})$-firm based QoS for each stream in terms of dynamic failure and delay at the given resource.

\subsection{DBP Outline}

DBP was firstly put forward in [4, as a dynamic priority assignment mechanism for streams with $(\mathrm{m}, \mathrm{k})$-firm constraint in a MIQSS model, and targeted primarily at loss tolerant, real-time applications like multimedia.

The basic idea of DBP algorithm is quite simple and straightforward: the closer the stream is to a failure state the higher its priority is. A failure state occurs when the stream's (m,k)-firm requirement is transgressed, i.e., there is more than $\mathrm{k}$ - $\mathrm{m}$ deadline misses within the last $\mathrm{k}$-length window.

So for each stream $\tau$ with $(\mathrm{m}, \mathrm{k})$-firm constraint, the priority is assigned based on the number of consecutive deadline misses that will lead the stream to violate its $(\mathrm{m}, \mathrm{k})$-firm constraint. This number of deadline misses is referred to as distance to fall into a failure state from the current state. Examining the recent history of $\tau$, one can do the evaluation of the distance. The key to dealing with it is the k-sequence. If the same distance occurs, Earliest Deadline First (EDF) will be adopted as adjunctive scheme.

The $\mathrm{k}$-sequence is a word of $\mathrm{k}$ bits ordered from the most recent to the oldest packet in which each bit keeps memory of whether the deadline is missed (bit= 0 ) or met (bit=1). In this paper, the leftmost bit represents the oldest. Each new arriving packet causes a shift of all the bits towards left, the leftmost exits from the word and is no longer considered, while the rightmost will be 1 if the packet has met its deadline (i.e. it has been served before its deadline) or 0 otherwise.

The priority is assigned by DBP to a packet at a given instant according to the distance of the current k-sequence to a failure state. By adding consecutive 0 s to the right side of k-sequence, we can evaluate the distance easily until a 
failure state happens. If a stream has already been in a failure state (i.e., less than $\mathrm{m} 1 \mathrm{~s}$ in the k-sequence), the highest priority is assigned.

Formally, according to [4], priority is evaluated as follows. Let $s_{j}=\left(\delta_{i-k_{j}+1}^{j}\right.$, $\left.\ldots, \delta_{i-1}^{j}, \delta_{i}^{j}\right)$ denotes the state of the previous $\mathrm{k}$ consecutive packets of $\tau_{j}, l_{j}(n, s)$ denotes the position (from the right) of the $n^{\text {th }}$ meet (or 1 ) in the $s_{j}$, then the priority of the $(i+1)^{t h}$ packet of $\tau_{j}$ is given by:

$$
\Omega_{i+1}^{j}=k_{j}-l_{j}\left(m_{j}, s_{j}\right)+1
$$

We note that if there are less than $\mathrm{n} 1 \mathrm{~s}$ in $s, l_{j}(n, s)=k_{j}+1$, the highest priority $(\Omega=0)$ will be assigned, this is normal as the stream is in a failure state.

Example1: a stream $\tau_{1}$ with $(3,5)$-firm constraint, current k-sequence is 11011 , we can get $l_{1}\left(3, s_{1}\right)=4$ and $\Omega_{i+1}^{1}=5-4+1=2$. If the current k-sequence is 10000 , then $l_{1}\left(3, s_{1}\right)=5+1$, so $\Omega_{i+1}^{1}=0$.

\subsection{Drawbacks of DBP}

Although DBP is more effective to guarantee $(\mathrm{m}, \mathrm{k})$-firm constraint, there are still some drawbacks. The first one is that it only uses the distance to fall into a failure state of $\mathrm{k}$-sequence whereas the whole richer information of " 0,1 " distribution in $\mathrm{k}$-sequence is neglected. In order to explain this problem, it is enough to consider different k-sequences with (2,5)-firm constraint: 11100 from $\tau_{1}$ and 11001 from $\tau_{2}$. They have the same distance $(\Omega=2)$. If they arrive at the same time, according to the DBP, EDF algorithm is default adjunctive scheduling scheme. But is EDF optimal to deal with such condition?

It appears that 11100 is less robust than 11001. For example, after a successful service of both, these two k-sequences become 11001 $(\Omega=2)$ and $10011(\Omega=4)$ respectively.But this is not necessary that we should firstly serve the next packet from $\tau_{1}$ even if it has earlier deadline. In fact, how to use such information will depend on what we would like to optimize. Maybe it is more complicated to set the priority and get the optimal objection function.

Another shortcoming of DBP is that it assigns priorities considering only one stream source without taking into account the parameters of other streams sharing the same server, which results in local priority that not global one and may lead to "priority inversion" phenomena. Improved algorithms to overcome the problem have been proposed in [6] [7].

\section{Extended Distance-Based Priority Scheduling}

One of the possible solutions to explore the ' 0,1 ' distribution in k-sequence is found when a stream falls in a failure state. Any packet in a failure stream has the same DBP value $(\Omega=0)$ but the distance to exit a failure state may be different. Let's take again the (2,5)-firm constraint as an example, DBP assigns the same priority to the following different k-sequences in failure states: 00001 and 10000, but to exit the failure state, 00001 needs only one more 1 whereas 
10000 needs two consecutive 1s. Especially in heterogeneous system, the streams have different $(\mathrm{m}, \mathrm{k})$-firm constraints, the above situation will occur frequently, so it is necessary to consider factor of ' 0,1 ' distribution.

Based on the information in k-sequence, including the ' 0,1 ' distribution, we propose to extend the notion of distance to failure state by introducing the notion of distance to exit failure state.

For each stream with $(\mathrm{m}, \mathrm{k})$-firm in a failure state, the priority is assigned based on the number of consecutive deadlines met that makes the stream back to meet its $(\mathrm{m}, \mathrm{k})$-firm constraint. The number of necessary consecutive deadlines met referred to as distance to exit a failure state from the current state.

The distance to exit a failure state is thus the number of consecutive $1 \mathrm{~s}$ adding to the right side. Formally, given a stream $\tau_{j}$ with constraint parameter $m_{j}$ and $k_{j}$ in a failure state, and let $s_{j}=\left(\delta_{i-k_{j}+1}^{j}, \ldots, \delta_{i-1}^{j}, \delta_{i}^{j}\right)$ be its current k-sequence. Define $\tilde{l}_{j}(n, s)$ as the position (from the right side) of the $n^{t h}$ miss in the state of $s_{j}$, so the distance to exit a failure state of stream is given by equation (2):

$$
\Phi_{i+1}^{j}=k_{j}-\tilde{l}_{j}\left(k_{j}-m_{j}+1, s_{j}\right)+1
$$

Example2: $\Phi=2$ for 100011 with (4,6)-firm constraint and $\Phi=1$ for 00011 with $(3,5)$-firm constraint.

In a successful state, priority is assigned according to DBP, while in a failure state priority is assigned by equation (2), in case of priority equality, EDF is adopted, which is referred as E_DBP. As discuss above, the definition of distance to exit a failure state is a symmetrical notion of distance to a failure state, just as if we look at in a mirror, so the negative logic is applied. It is supposed that if the packet in the stream with smaller $\Phi$ value gets higher priority, and a successful service for a packet adds 0 to the right of $\mathrm{k}$-sequence, which will make the stream get more chance to be in failure states like DBP to guarantee successful states. But in fact, a packet is serviced by its deadline, 1 will be added to right of k-sequence, which will be more easy to a exit failure state. So it is reasonable to assign higher priority to the packet from the stream having smaller $\Phi$ value. The detailed priority assigning process can be described as follows.

E_DBP precedence among all being selected packets

- If all streams are in successful states, the smaller DBP value, the higher priority. If DBP value $\Omega$ is the same, EDF is adopted.

- If just only stream $\tau_{j}$ is in a failure state, others are in successful states, the packet in the stream $\tau_{j}$, gets higher priority.

- If many streams are in failure states at the same time, the smaller $\Phi$, the higher priority. If $\Phi$ value is the same, EDF is adopted.

- For all cases, if the same deadline, then FIFO.

\section{Simulation Result}

The new proposed algorithm, E_DBP is compared with DBP through simulation examples given in 4]. QoS in terms of probability of dynamic failure and delay is 
taken as performance metric. In the results that follow, two packets generation patterns are considered: Poisson and burst. In a Poisson stream, packet interarrival times are exponentially distributed. A burst source alternates between $\mathrm{ON}$ and $\mathrm{OFF}$ states. When in the ON state, packets are generated periodically. No packets are generated when source is in the OFF state. The durations of the $\mathrm{ON}$ and the OFF states are exponentially distributed with averages $O N_{\text {ave }}$ and $O F F_{\text {ave }}$ respectively. Such a stream is often used to model a stream of voice sample in a conversation [8. We firstly consider the case where all streams in the system have the same timing requirements. We also assume that only the packets that meet their deadline are serviced, which means that drop policy is enabled. Simulation adopts software OPNET8.0.c Modeler. Time duration of all projects is 20000. There we define $\sum_{i=1}^{n} \frac{C_{i}}{E\left(T_{i}\right)}$ as average system Load and $\sum_{i=1}^{n} \frac{m_{i} C_{i}}{k_{i} E\left(T_{i}\right)}$ as average system $(\mathrm{m}, \mathrm{k})$-Load, where $E\left(T_{i}\right)$ is the mean inter-arrival time. The initial k-sequence is $\underbrace{11 \ldots 1}_{k_{i}}$ for a stream with windows $k_{i}$.

\subsection{Evaluation of Dynamic Performances}

Poisson Streams The data in the left column in Table1 1 shows the probability of dynamic failure in one system with $(3,4)$-firm constraint. The system consists of five streams. All packets require a constant service time. Service deadlines are set equal to five times the packet service time. The packet inter-arrival time is exponentially distributed and the overall average load is varied from 1.0-2.0 by changing inter-arrival time. As a result, it is shown that new scheme E_DBP can reduce the probability of dynamic failure, especially when it is overloaded. The maximum reduced percent is $9.3 \%$ in this case when load equals to 2.0 .

The above system considers that all streams have the same deadline requirement with $(3,4)$-firm. The middle column in the Table 1 shows the results for the heterogeneous system in which steams have different deadline requirement. The system consists of five systems with (9,10)-firm, (3,4)-firm, (1,2)-firm, (1,3)-firm, $(1,4)$-firm constraint, respectively. The packet service time, arrival pattern, and the deadline in this system are like those for the stream examined in the first example. The arrival rates of the packets are adjusted to get an average system load from 1.3-2.3. Simulation result shows that even at load 1.4-1.6, there is a little abnormal behavior that E_DBP is slightly worse than DBP (1.0\% to $2.3 \%$ increase). But there is still a strong trend that E_DBP can reduce the probability of dynamic failure, especially about $8.6 \%$ at load 2.3 .

Burst Streams The data in the right column in Table 1 of probability of dynamic failure in a system with five burst streams. The ON and OFF periods of each stream are exponentially distributed with $O N_{\text {ave }}=50$ and $O F F_{\text {ave }}=100$. The offered peak load of a stream is therefore three times the average load. When in the ON state, a stream generating one packet is 5 periodically. The deadlines are set to twice the generation period. Overall load varies by changing packet service time. We find that E_DBP is better than DBP to guarantee the 
Table 1. Probability of dynamic failure in Poisson streams with same constraint, heterogeneous system and burst streams

\begin{tabular}{|c|c|c|c|c|c|c|c|c|c|c|c|}
\hline \multicolumn{4}{|c|}{ Poisson Stream } & \multicolumn{4}{|c|}{ Heterogeneous system } & \multicolumn{4}{|c|}{ Burst streams } \\
\hline $\begin{array}{l}\text { Avg } \\
\text { Load }\end{array}$ & $\mathrm{DBP}$ & E_DBP & $\begin{array}{c}\% \\
\mathrm{Rd}\end{array}$ & $\begin{array}{c}\text { Avg } \\
\text { Load }\end{array}$ & $\mathrm{DBP}$ & E_DBP & $\begin{array}{c}\% \\
\mathrm{Rd}\end{array}$ & $\begin{array}{l}\text { Avg } \\
\text { Load }\end{array}$ & $\mathrm{DBP}$ & E_DBP & $\begin{array}{c}\% \\
\mathrm{Rd}\end{array}$ \\
\hline 1.0 & 0.055 & 0.055 & ـ & 1.3 & 0.032 & 0.032 & - & 0.5 & 0.000 & 0.000 & - \\
\hline 1.1 & 0.096 & 0.095 & 0.8 & 1.4 & 0.053 & 0.054 & -2.3 & 0.6 & 0.006 & 0.006 & 3.3 \\
\hline 1.2 & 0.156 & 0.154 & 1.3 & 1.5 & 0.083 & 0.084 & -1.6 & 0.7 & 0.031 & 0.031 & - \\
\hline 1.3 & 0.229 & 0.223 & 2.4 & 1.6 & 0.115 & 0.117 & -1.0 & 0.8 & 0.078 & 0.075 & 4.0 \\
\hline 1.4 & 0.311 & 0.299 & 3.9 & 1.7 & 0.157 & 0.157 & 0.3 & 0.9 & 0.150 & 0.145 & 3.3 \\
\hline 1.5 & 0.398 & 0.378 & 5.0 & 1.8 & 0.200 & 0.198 & 0.8 & 1.0 & 0.227 & 0.213 & 6.0 \\
\hline 1.6 & 0.481 & 0.449 & 6.5 & 1.9 & 0.245 & 0.240 & 1.8 & 1.1 & 0.296 & 0.270 & 8.7 \\
\hline 1.7 & 0.557 & 0.514 & 7.8 & 2.0 & 0.293 & 0.284 & 3.1 & 1.2 & 0.371 & 0.337 & 9.1 \\
\hline 1.8 & 0.623 & 0.569 & 8.7 & 2.1 & 0.339 & 0.323 & 4.8 & 1.3 & 0.449 & 0.409 & 8.8 \\
\hline 1.9 & 0.675 & 0.612 & 9.2 & 2.2 & 0.381 & 0.362 & 6.6 & 1.4 & 0.509 & 0.472 & 7.4 \\
\hline 2.0 & 0.716 & 0.649 & 9.3 & 2.3 & 0.431 & 0.394 & 8.6 & 1.5 & 0.556 & 0.509 & 8.4 \\
\hline
\end{tabular}

$(\mathrm{m}, \mathrm{k})$-firm constraint and substantially reduce probabilities of dynamic failure obviously with average load varied from 0.5-1.5. At load 1.2, there is maximum reduction percent $9.1 \%$. It is obvious that the probability of dynamic failure is higher in burst case than in Poisson case at the same average load, because the peak load is heavier and the packets are more concentrated in the burst case.

\subsection{Delay Analysis}

Delay is the time interval between the departures of packet from the source to the arrival at the destination. This is usually referred to as end-to-end delay. In MIQSS model, delay just only means the queue delay. Delay is an important parameter of QoS. Many real-time applications such as voice over IP (VoIP), video-conference, and tele-medicine require guarantees on delay and packet loss. These applications are usually sensitive to delay and loss-tolerance. Smaller delay will make media stream more smoothly. This statistic represents instantaneous measurements of packet waiting times in the queue of server, and delay of all discarded packets is not calculated in this statistic. The simulation results also through the above three examples reveal that E_DBP can reduce the delay than DBP at different degree when $(\mathrm{m}, \mathrm{k})$-load is varied. At some appropriate load duration, queue delay is decreased more effectively, but some light load, it is not so significant. The third example is burst stream, the packets are more concentrated at the ON state, so at a lighter $(\mathrm{m}, \mathrm{k})$-load in burst case, we can still get the similar result with the Poisson stream cases. For the delay in the burst case, it fluctuates acutely because of concentration of packets. 

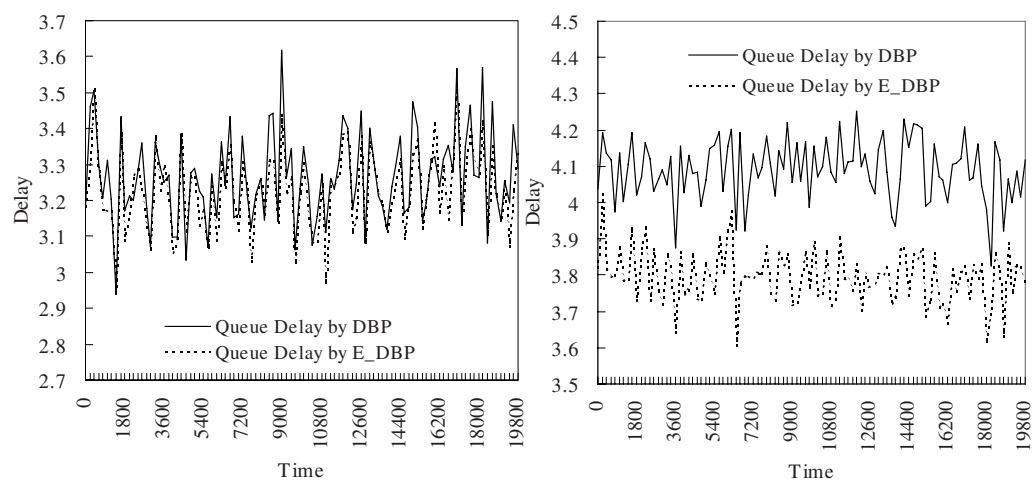

Fig. 1. Queue delay comparison of E_DBP and DBP for Poisson streams with $(3,4)$-firm at $(\mathrm{m}, \mathrm{k})$-load equals to 1 and 1.5

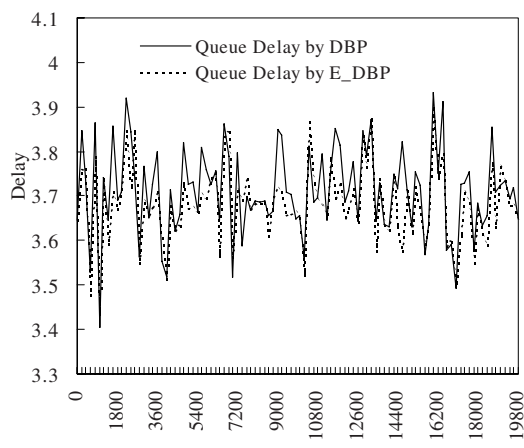

Time

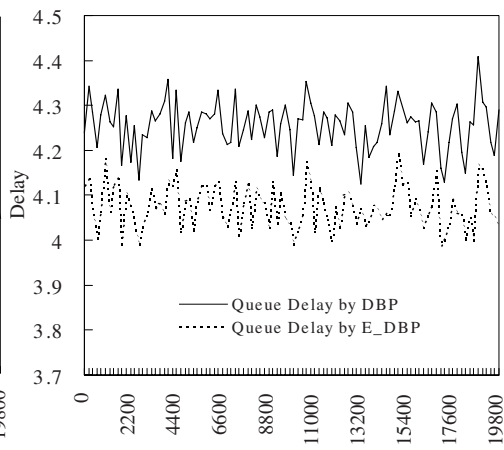

Time

Fig. 2. Queue delay comparison of E_DBP and DBP for heterogeneous system at $(\mathrm{m}, \mathrm{k})$-load equals to 1 and 1.5
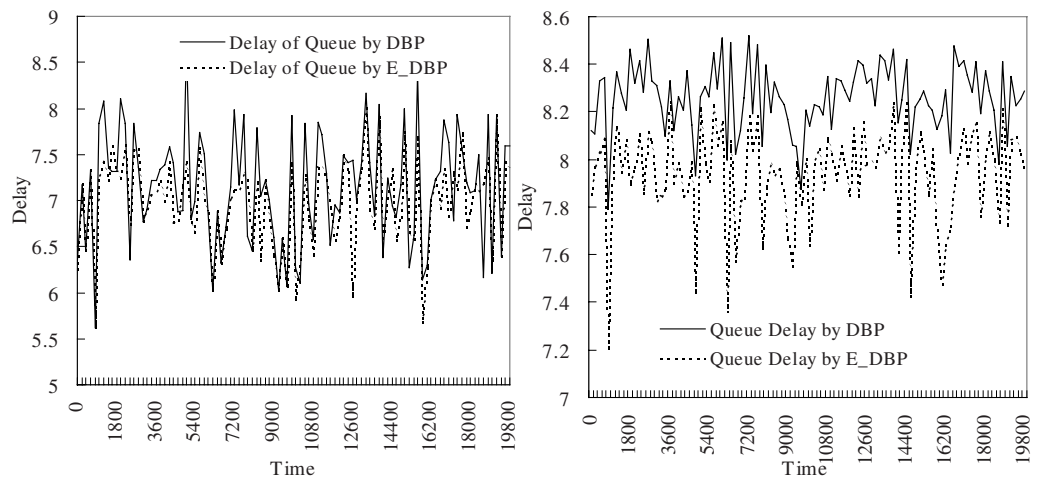

Fig. 3. Queue delay comparison of E_DBP and DBP for burst stream $(3,4)$-firm at $(\mathrm{m}, \mathrm{k})$-load equals to 0.5 and 1 


\section{Conclusions}

The main original contributions of this paper are:

- Point out the drawbacks of the classic DBP when it is applied to a more general real-time context and corresponding possible solutions.

- Propose E_DBP to correct DBP by taking the distribution of " 0,1 " in ksequence into account when stream is in a failure state, and give the equation to calculate the distance to exit failure state.

- Show that E_DBP can get lower probability of dynamic failure and smaller queue delay than classic DBP, which is validated by various cases through simulation based on OPNET 8.0.c.

This improvement is made with a very low computing cost or complexity, only to check minimum 1 s, which needs to be added to the right position. Furthermore, this new computing is only needed when the stream is in a failure state. In this sense, our algorithm is interesting in guaranteeing $(\mathrm{m}, \mathrm{k})$-firm QoS in network scheduling. Furthermore, WFQ and RED combined with (m,k)-firm constraint also may be an interesting work.

\section{Acknowledgements}

The authors thank the anonymous reviewers for their valuable comments that improve the quality of this paper and financial support of Sino-France Advanced Research Program(No:PRA SI03-02) and NSFC(No:60203030).

\section{References}

[1] Mittal, A., Manimaran, G., Siva Ram Murthy, C.: Integrated Dynamic Scheduling of Hard and QoS Degradable Real-Time Tasks in Multiprocessor Systems. Journal of Systems Architecture. 46 (2000) 793-807

[2] El-gendy, M.A., Bose, A., Shin, K.G.: Evolution of the Internet QoS and Support for Soft Real-Time Applications. Proceedings of IEEE. 91 (2003) 1086-1104

[3] Peha, J.M., Tobagi, F.A.: A Cost-Based Scheduling Algorithm to Support Integrated Services. In: Proceedings of IEEE INFOCOM., IEEE (1991) 741-753

[4] Hamdaoui, M., Ramanathan, P.: A Dynamic Priority Assignment Technique for Streams with (m,k)-Firm Deadlines. IEEE Transactions on Computers. 44 (1995) 1443-1451

[5] Bernat, G., Burns, A., Llamosi, A.: A Weakly Hard Real-Time Systems. IEEE Transaction on Computers. 50 (2001) 308-321

[6] Poggi, E.M., Song, Y.Q., Koubaa, A., Wang, Z.: Matrix-DBP for (m,k)-Firm RealTime Guarantee. In: Proceedings of Real-Time and Embedded System., Paris (2003) 457-482

[7] Chen, J.M., Song, Y.Q., Wang, Z., Sun, Y.X.: Equivalent Matrix-DBP for (m,k)Firm Deadline. In: Proceedings of IEEE International Symposium on Industrial Electronics. (2004) 675-680

[8] Saleh, M.A., Habib, I.W., Saadawi, T.N.: Simulation Analysis of A Communication Link with Statistically Multiplexed Bursty Voice Sources. IEEE Journal on Selected Areas in Communication. 11 (1993) 432-442 